\title{
BMJ Open Multimodal neuronavigation-guided precision bypass in adult ischaemic patients with moyamoya disease: study protocol for a randomised controlled trial
}

Junlin Lu, ${ }^{\oplus}$ Yahui Zhao, ${ }^{1} \mathrm{Li} \mathrm{Ma},{ }^{1}$ Yu Chen, ${ }^{1}$ Mingtao $\mathrm{Li},{ }^{1}$ Xun Ye, ${ }^{1,2}$ Rong Wang, ${ }^{1,2}$ Xiaolin Chen, ${ }^{1,2}$ Yuanli Zhao ${ }^{1,2}$

To cite: Lu J, Zhao Y, Ma L, et al. Multimodal neuronavigationguided precision bypass in adult ischaemic patients with moyamoya disease: study protocol for a randomised controlled trial. BMJ Open 2019;9:e025566. doi:10.1136/ bmjopen-2018-025566

- Prepublication history for this paper is available online. To view these files, please visit the journal online (http://dx.doi. org/10.1136/bmjopen-2018025566).

Received 21 July 2018 Revised 15 December 2018 Accepted 20 December 2018

Check for updates

(C) Author(s) (or their employer(s)) 2019. Re-use permitted under CC BY-NC. No commercial re-use. See rights and permissions. Published by BMJ.

${ }^{1}$ Department of Neurosurgery, Beijing Tiantan Hospital , Capital Medical University, Beijing,

China

${ }^{2}$ Department of Neurosurgery, PekingUniversity International Hospital, Beijing, China

Correspondence to

Professor Yuanli Zhao;

zhaoyuanli@126.com

\section{ABSTRACT}

Introduction Revascularisation surgery is an important treatment of moyamoya disease (MMD). Several general methods of revascularisation had been used: direct, indirect and combined techniques. However, there had been no reports about the criteria of recipient arteries selection in bypass surgery for MMD. Surgeons usually choose the recipient arteries by their own experiences. Their choices of the recipient arteries are various and may contribute the different outcome of patients. The purpose is to identify utility and efficacy of precision bypass guided by multimodal neuronavigation of MMD in a prospective randomised controlled trial.

Method and analysis This study is a prospective randomised controlled clinical trial. This study will enrol a total of 100 eligible patients. These eligible patients will be randomised to the empirical bypass group and the multimodal neuronavigation-guided precision bypass group in a 1:1 ratio. Patient baseline characteristics and MMD characteristics will be described. In the multimodal neuronavigation-guided group, the blood velocity and blood flow of the recipient arteries will be identified. Surgical complications and outcomes at pretreatment, post-treatment, at discharge and at 3 month, 6 month, 12 month and end of trial will be analysed with CT perfusion, MRI, digital subtraction angiography, modified Rankin Scale, National Institute of Health Stroke Scale and modified Barthel Scale. This trial will determine whether multimodal neuronavigation-guided precision bypass is superior to empirical bypass in patients with MMD and identify the safety and efficacy of multimodal neuronavigation-guided precision bypass.

Ethics and dissemination The study protocol and written informed consent were reviewed and approved by the Clinical Research Ethics Committee of Peking University International Hospital. Study findings will be disseminated in the printed media. The study started in August, 2018 and expected to be completed in December, 2020.

Trial registration number NCT03516851; Pre-results.

\section{BACKGROUND}

Moyamoya disease (MMD) is an uncommon cerebrovascular disorder characterised by
Strengths and limitations of this study

- To our knowledge, this trial is the first prospective randomised controlled clinical trial comparing 'empirical' combined revascularisation surgery with 'precise' combined revascularisation surgery to evaluate the role of 'precise' combined revascularisation surgery in the treatment of moyamoya disease (MMD).

- The blinding with outcome assessors who are not involved in patient management helps to minimise bias associated with outcome evaluation.

- Implementing digital subtraction angiography and MRI (including diffusion weighted imaging and arterial spin labelling) followed by a radiological diagnosis prior to enrolment in all adult patients with MMD.

- Single centre original study with thorough recording of data on all evaluated patients may not exclude all the selection bias.

progressive occlusion of the internal carotid artery (ICA) terminus and its main branches, leading to compromised cerebral perfusion and recurrent ischaemic strokes. ${ }^{1}$ Epidemiological features of MMD have been reported in various regions during the past three decades. The observations have shown regional ${ }^{1-3}$ and ethnic differences ${ }^{45}$ in incidence, prevalence and patient characteristics. MMD is estimated to have a prevalence of $1.61-3.92$ per 100000 in China. ${ }^{67}$ Clinical features of MMD differ substantially between children and adults. The main clinical manifestation of MMD is transient ischaemic attack (TIA) or cerebral infarction in children, whereas about half of adult patients develop intracranial haemorrhage, and half develop TIA or cerebral infarction, or both. ${ }^{8}$

Ample evidences show that there are no effective medical therapies for MMD, 
revascularisation surgery has played an important role in treatment of MMD. ${ }^{19}$ Several general methods of revascularisation had been used: direct, indirect and combined techniques. Three large long-term follow-up studies showed a good safety profile for surgical treatment. Patients with MMD have a higher risk of neurological events during perioperative period and these treatment-related complications include stroke, infection and intracranial haemorrhage. During the postoperative 30 days, the postoperative complications occurrence ranged from $4.7 \sim 32.7 \%$, and after the first month the risk decrease fairly. ${ }^{10-15}$ It is reported that the recurrent stroke events were observed in 5.5 8.2\% of patients during the follow-up period. ${ }^{11} 1315$ Moreover, patients with postoperative complications had worse neurological status at discharge. $^{15}$

Many focuses located on the risk of subsequent stroke or haemorrhage even with surgical revascularisation and regular medical therapy in MMD patients. A retrospective, single-centre, cohort study showed most subsequent ischaemic events appeared in the first 2 years after surgery. The Kaplan-Meier estimated stroke risk was $10.1 \%$ and $12.7 \%$ in the first 2 years and 5 years after surgery for all patients treated with surgical revascularisation, approximated $10.1 \%$ incidence of mortality or TIA or stroke at 1 year after surgery in 802 patients with the MMD. ${ }^{16}$ Incidence of rebleeding range from $12.5 \sim 20 \%$ of patients with onset haemorrhage symptom who underwent revascularisation surgery, while with the conservative treatment the rebleeding was estimated occurrence of $6.8 \%-65 \% .{ }^{17-21}$

To our best knowledge, there are no reports concerning the criteria for recipient artery selection in bypass surgery for MMD. In clinical practice, superficial temporal artery to middle cerebral artery (STA-MCA) anastomosis was the most preferred technique, ${ }^{22}$ sometimes, anastomoses of STA to the branch of the anterior cerebral artery (ACA) might also be adopted when ACA territory showed significantly compromised perfusion. ${ }^{23} 24$ There may be less variability in the size and site of craniotomy, which causes less variability in the area of revascularisation. Due to the imprecise revascularisation area, some patients might still suffer from TIA after revascularisation surgery. ${ }^{13} 16$ Moreover, there may be multiple alternative recipient arteries within the centre of the craniotomy. Different selection of recipient arteries may lead to discrepancies in the patient outcome. However, how to precisely choosing the detail segment of MCA or ACA as recipient artery remains uncertain.

Arterial spin labelling (ASL) offers a noninvasive method for quantifying cerebral blood flow (CBF) by using magnetically labelled arterial blood water as an endogenous tracer and has been increasingly applied to imaging studies on neurological and psychiatric diseases. ${ }^{25-27}$ Small case series have reported that ASL is a valuable tool for evaluating haemodynamic changes after MMD.$^{28}$ Ample evidences also suggest that ASL has good agreement with other measures of cerebral perfusion assessment. ${ }^{29-31}$ Some reports mentioned that intraoperative indocyanine green (ICG) video angiography combined with haemodynamic parameter analysis obtained by Flow 800 software is useful for intraoperative monitoring of regional CBF in cerebrovascular disease. ${ }^{32-34}$ It can provide detailed images for vascular architecture, flow direction and blood flow transit, which provides real-time cerebral flow velocity and superficial perfusion of local brain. ${ }^{35}$ These techniques could potentially influence the evaluations of surgery. Moreover, standard tasks and a definitive analysis of ASL data are required to be achieved before drawing a definitive conclusion. Therefore, this prospective, exploratory, randomised controlled trial will identify the safety and efficacy of multimodal neuronavigation-guided precise direct bypass microsurgery of MMD.

\section{STUDY OBJECTIVE}

The key purpose of this study is to determine whether multimodal neuronavigation-guided direct bypass surgery is superior to empirical direct bypass procedure for preventing the postoperative strokes or other bad outcomes in patients with MMD. We will assess the safety and efficacy of multimodal neuronavigation-guided precise direct bypass surgery.

\section{METHODS AND ANALYSIS \\ Study designs}

This will be an investigator-initiated and prospective, exploratory, randomised controlled trial. Eligible patients with ischaemic MMD undergoing surgery will be randomised to the empirical direct bypass procedure group and the multimodal neuronavigation-guided precise direct bypass surgery group in a 1:1 ratio. The study protocol was performed at Peking University International Hospital. All patients with MMD will be treated by the same operator (RW). A diagram of the protocol is presented in figure 1.

\section{Study participants}

All patients who meet the clinical criteria will undergo MRI, perfusion CT (CTP) and digital subtraction angiography (DSA). Patient baseline characteristics (age of randomisation, sex and neurological function) and MMD characteristics (Suzuki stage, moyamoya vessels, posterior cerebral artery involvement and collateral pathways) will be provided. Treatment decision is made based on previous reports. ${ }^{36}$ Surgical treatment is preferably considered for patients who have a history of infarctions or TIA. Treatment modalities for MMD and randomised methods will be informed to the patient and family. Eligible patients will be included before the written informed consent is obtained. These patients can withdraw from the study at any time.

\section{Inclusion criteria}

- Ages eligible for study ranges from 18 years to 60 years (adult) 

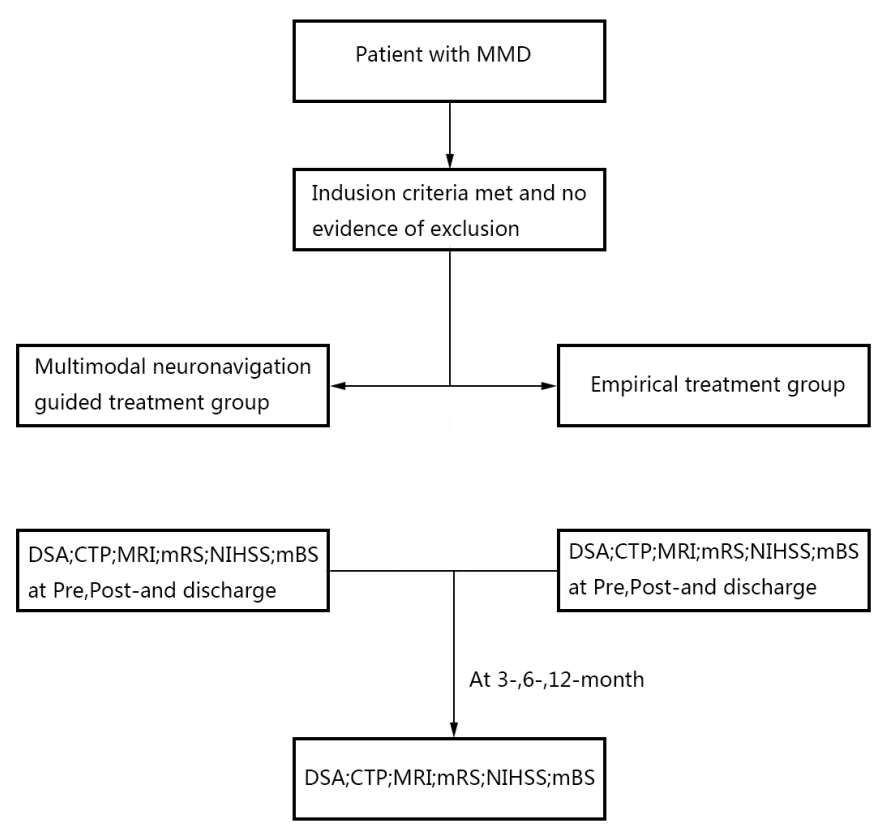

Figure 1 Diagram of the study protocol. CTP, perfusion CT; DSA, digital subtraction angiography; mBS, modified Barthel Scale; MMD, moyamoya disease; mRS, modified Rankin Scale; NIHSS, National Institute of Health Stroke Scale.

- Independent in activity of daily living (the modified Rankin Scale (mRS) 0-2)

- At least 1 month since the most recent ischaemic stroke

- The neurological deficit must be stable for more than 6 weeks

- DSA demonstrating progressive stenosis or occlusion in the terminal portion of the ICA and/or the initial portion of the anterior or middle cerebral arteries

- DSA demonstrating formation of abnormal collateral networks (moyamoya vessels) at the base of the brain, mainly in the region of thalamus and basal ganglia

- DSA demonstrating the vasculopathy appeared unilaterally or bilaterally

- Competent to give informed consent

- Accessible and reliable for follow-up

\section{Exclusion criteria}

- Other diseases (such as ICA stenosis, ICA dissection, atrial fibrillation or intracranial atherosclerosis) probably causing ischaemic strokes.

- Not independent in activity of daily living (the mRS 3-5).

- Moyamoya syndrome concomitant with other hereditary or autoimmune diseases (Grave's disease, type I diabetes mellitus, type I neurofibromatosis and so on).

- Patient whose initial onset was marked by ischaemia but subsequently suffered from intracranial haemorrhage.
- Emergent evacuation of intracerebral haematoma damaging superficial temporal artery or cortical artery.

- Prior revascularisation surgery for MMD.

- Good collateral networks formed by spontaneous anastomosis between extracranial and intracranial vessels before surgery

- Life expectancy $<1$ year.

- Pregnancy and breast feeding.

- Unstable angina or myocardial infarction with recent 6 months.

- Blood coagulation dysfunction.

- Allergic to iodine contrast agent.

- Abnormal liver function (alanine transaminase and/or aspartate aminotransferase $>3$ times of normal range).

- Serum creatinine $>3 \mathrm{mg} / \mathrm{dL}$.

- Poorly controlled hypertension (systolic blood pressure (BP) $>160 \mathrm{~mm} \mathrm{Hg}$, diastolic $\mathrm{BP}>100 \mathrm{~mm} \mathrm{Hg}$ ).

- Poor glucose control (fasting blood glucose $>16.7 \mathrm{mmol} / \mathrm{L})$.

- Concurrent participation in any other interventional clinical trial.

- Patients refused to participate in the study.

\section{Randomisation and blinding}

Eligible patients will be randomised to the empirical direct bypass procedure group and the multimodal neuronavigation-guided precise direct bypass surgery group in a 1:1 ratio. Stratified block randomisation and minimisation methods are employed. Stratification factors include mRS and the Suzuki stage. It is impossible to blind the participants and surgeons, and only the outcome assessors who are not involved in patient management are blinded. The allocation sequence will be generated by an independent third party of the China National Clinical Research Center for Neurological Diseases, and patients treated with multimodal neuronavigation-guided precise direct bypass surgery will be assigned by the third party. To prevent bias, the patient randomisation process will be performed by the third party.

\section{Empirical direct bypass surgery group}

Patients in the empirical direct bypass surgery group will receive cerebral angiography, perfusion CT and MRI (including ASL and diffusion weighted imaging sequences) before surgical treatment. The craniotomy side will be selected based on symptomatic and haemodynamically affected hemisphere. The craniotomy will be performed within the cerebrovascular team. Empirical direct bypass surgery includes dissection of the superficial temporal artery from the adjacent tissue, temporary clipping of the recipient artery and anastomosed the superficial temporal artery to the recipient artery. ${ }^{22-24}$ Any techniques for motor evoked potential and somatosensory evoked potential can be used for intraoperative monitoring. Intraoperative indocyanine green videoangiography (ICG-VA) can also be used to determine the patency of the bypass vessel as needed. 
Multimodal neuronavigation-guided precise direct bypass surgery group

Patients randomised to the multimodal neuronavigation-guided precise direct bypass surgery group will receive cerebral angiography, perfusion CT and MRI before surgery. Arterial spin labelling (ASL), diffusion weighted imaging (DWI) and structure sequence (T2 FLAIR) will be performed ${ }^{37-39}$ ASL and DWI are automatically fused with structure sequence (T2 FLAIR) images. The data of CBF will be imported to the neuronavigation system (Medtronic, Inc) preoperatively.

Surgeons can select craniotomy side precisely based on where cerebral ischaemia (misery perfusion) exist. With the brain cortex exposed after craniotomy, an initial ICG-VA will be performed and ICG images were analysed with FLOW 800 software (ZEISS, Germany). From the colour maps of FLOW 800 surgeons assessed the microvascular transit time (MVTT) of the different candidates for recipient artery and chose the artery with the highest MVTT as our first candidate. The treatment planning station will be situated based on the multimodal neuronavigation data. The ASL and structure sequence data sets were applied to the neuronavigation system (Stealth Station; Medtronic, Minneapolis, Minnesota, USA) and the fusion process was carried out with these images. The perfusion of candidates for recipient artery for the surgery can be evaluated in these consecutive fusion images. From them, surgeons select the artery with the highest MVTT (lowest velocity) that was located on or near the cortex where perfusion was markedly decreased as the target. Then a direct bypass procedure will be performed just like in the empirical direct bypass surgery group.

The multimodal-guided precise direct bypass surgery will be discontinued if there is a medical necessity or at the request of the participant. This could occur if the participant withdraws from the study at any time and for any reason. Investigators will collect data in detail at the time of study randomisation. Adherence to treatment allocation will be monitored in the monthly reports, which originate from the data monitoring centre (China National Clinical Research Center for Neurological Diseases). All surgical techniques and equipment will be provided as needed in both treatment groups. Any concomitant medication in the context of the study is permitted.

\section{Outcome measures}

The primary outcome is the occurrence rate of strokes or death and ipsilateral intracranial haemorrhage of any kind within 30 days postsurgery. Other outcomes are the postoperative haemodynamic changes, postoperative complications, neurological function and retreatment. haemodynamic changes include changes in moyamoya vessels,perfusion area of the feeding artery according to the postoperative cerebral angiography and CBF assessed by postoperative CTP and ASL. Surgical complications include infarctions on the contralateral side, TIA and all kinds of adverse events related to surgery. Patient baseline neurological function and outcomes at pretreatment, post-treatment, at discharge and at 3 month, 6 month and 12 month follow-ups are measured using the mRS, National Institute of Health Stroke Scale (NIHSS) and the modified Barthel Scale. A schematic diagram is showed in figure 1 . A mRS of $0-2$ is identified as a favourable outcome, and a score of 3-6 as an unfavourable outcome. A NIHSS of $0-14$ is identified as a favourable outcome, and a score of 15-42 as an unfavourable outcome. A modified Barthel Scale of 61-99 identified as a favourable outcome, and score of 0-60 as an unfavourable outcome.

\section{Sample size}

Because this trial is a prospective, exploratory, randomised controlled trial, there is no previous data for multimodal neuronavigation-guided precise bypass procedure, the sample size for this trial can't be calculated from the formula. Finally, 100 patients are randomised (1:1) to two groups, 50 of them will undergo direct bypass guided by multimodal neuronavigation, while others will undergo empirical direct bypass procedure.

\section{Statistical analysis}

All analyses will be based on the intent-to-treat principle using the full analysis set. Data are given as the mean and SD for continuous variables or frequency for categorical variables. Significances in clinical variables and outcomes between two groups are analysed using the t-test, $\chi 2$ test, Fisher's exact test or the Mann-Whitney U test. Associations of variables will be identified using a univariate and multivariate analysis. Statistical analysis will be performed at the China National Clinical Research Center for Neurological Diseases.

\section{Data management}

Data are prospectively collected using an electronic case report form through a study website using a login and password. Neurological conditions and complications related to surgical procedure will be carefully monitored during the whole period of follow-up. These data safety will be monitored at the China National Clinical Research Center for Neurological Diseases during the clinical trial. A third party will review the progress of this study (eg, enrolment, site performance, meeting risk factor targets) as well as data on the safety of both treatment arms (eg, complications of multimodal neuronavigation-guided precise bypass surgery) to ensure that the study meets the highest standards of ethics and patient safety approximately every 3 months. Any serious adverse events will be reported to the Institutional Review Board of Peking University International Hospital. Recommendations for a review of risk and benefit will be forwarded to the principal investigators. The Institutional Review Board of the hospital shall obtain these interim results and make the final decision to terminate the trial.

\section{Duration of the study}

This study will enrol eligible 100 patients with MMD treated with cerebral revascularisation surgery in Peking University International Hospital. Study recruitment 
commenced in August 2018. The final patient recruitment will end in August 2020.

\section{Patient and public involvement}

The development of the research question and outcome measures were based on the patient-centred outcome of future patients gaining major benefit from the revascularisation surgery. Patients and the public were not directly involved in the design, recruitment or conduct of this pilot study. And the burden of the intervention was not assessed by patients themselves. The results of this study will be disseminated in peer-reviewed journals and at academic conferences. A summary of the study report will be written for patients through WeChat (a free messaging and calling application) account or group.

\section{ETHICS AND DISSEMINATION}

The Original Protocol for this prospective, exploratory, randomised controlled trial was registered in May 2018. The study protocol was performed at Peking University International Hospital and all the surgery will be performed by the same neurosurgeon to reduce surgery bias and multimodal neuronavigation bias. All patients will undergo MRI examinations during perioperative period. Therefore, patients with implanted medical devices will be excluded. And other unexpected risks of MRI examination will be monitored. A minor amount of brain shift exists in neuronavigation, ${ }^{40}$ but it does not influence the surgical procedure. This study adheres to good clinical practice and ethical principles described in the Declaration of Helsinki and 'Human Biomedical Research Ethical Issues and Policy Guidance'. Each eligible patient who wishes to participate in this study will be invited to give written informed consent. Professor Yuanli Zhao, Rong Wang and Xiaolin Chen are the principal investigators. Data interpretation and dissemination of study results will be managed under their direct supervision. The result will be disseminated in December 2020. The results of this study will be published in open-access and peer-reviewed journals and presented at relevant conferences.

\section{DISCUSSION}

Increasingly, through the provision of collateral pathway, surgical revascularisation is gaining acceptance as the most successful therapy to improve cerebral haemodynamics, and to reduce the risk of subsequent stroke for MMD. ${ }^{41}$ Direct bypass is useful to improve cerebral haemodynamics and to resolve ischaemic attacks immediately after surgery. ${ }^{24}$ However, careful management of patients is needed after direct bypass surgery because postoperative pronounced changes in cerebral haemodynamics might be more likely to induce neurological deficits, especially in patients with preoperative profound ischaemia. ${ }^{42}$ Currently, few report about recipient artery selection criterion for MMD bypass surgery. Neurosurgeons usually choose the recipient artery with their own experience. Their criteria are various and the effects of different recipient artery selection criterions remain uncertain. As the imaging advanced, ASL can be used as a perfusion method to detect the ischaemia area and intraoperative ICG angiography using FLOW800 software can be used to reflect the lower flow velocity and superficial perfusion. Combine two imaging data into the navigational system, which can help guide the precision direct bypass surgery of MMD. As the first prospective, exploratory, randomised controlled trial mainly focused on outcomes of MMD. We want to confirm that multimodal neuronavigation can safely be used for the precision direct bypass surgery of MMD, and multimodal neuronavigation-guided precision direct bypass microsurgery could improve the overall outcomes in patients with MMD.

Acknowledgements We acknowledge all patients evaluated for study enrolment and especially all participating patients, neurosurgeons, radiologists, anesthesiologists and nurses.

Contributors YuZ obtained research funding and was the principal investigator of the study. YaZ, LM, YC, ML and XY developed this study protocol. JL, XC, RW and YuZ all participated in the final design of the study.

Funding This study was supported by the Ministry of Science and Technology of China, National Key Technology Research and Development Program (2015BAl12B04); and National Natural Science Foundation of China (H0906 81571110 and H0906 81771234 to Y.L. Zhao; 81500995 to Xiaolin Chen).

Competing interests None declared.

Patient consent for publication Not required.

Ethics approval This final study protocol (No.201805) and written informed consent (No.201805) have been approved by the Clinical Research Ethics Committee of Peking University International Hospital in May, 2018. This amendment has been approved by the Clinical Research Ethics Committee.

Provenance and peer review Not commissioned; externally peer reviewed.

Open access This is an open access article distributed in accordance with the Creative Commons Attribution Non Commercial (CC BY-NC 4.0) license, which permits others to distribute, remix, adapt, build upon this work non-commercially, and license their derivative works on different terms, provided the original work is properly cited, appropriate credit is given, any changes made indicated, and the use is non-commercial. See: http://creativecommons.org/licenses/by-nc/4.0/.

\section{REFERENCES}

1. Kuroda S, Houkin K. Moyamoya disease: current concepts and future perspectives. Lancet Neurol 2008;7:1056-66.

2. Chiu D, Shedden P, Bratina P, et al. Clinical features of moyamoya disease in the United States. Stroke 1998;29:1347-51.

3. Kleinloog R, Regli L, Rinkel GJ, et al. Regional differences in incidence and patient characteristics of moyamoya disease: a systematic review. J Neurol Neurosurg Psychiatry 2012;83:531-6.

4. Uchino K, Johnston SC, Becker KJ, et al. Moyamoya disease in Washington State and California. Neurology 2005;65:956-8.

5. Graham JF, Matoba A. A survey of moyamoya disease in Hawaii. Clin Neurol Neurosurg 1997;99(Suppl 2):S31-5.

6. Chen PC, Yang SH, Chien KL, et al. Epidemiology of moyamoya disease in Taiwan: a nationwide population-based study. Stroke 2014;45:1258-63.

7. Miao W, Zhao PL, Zhang YS, et al. Epidemiological and clinical features of Moyamoya disease in Nanjing, China. Clin Neurol Neurosurg 2010;112:199-203.

8. Fukui M, Kono S, Sueishi K, et al. Moyamoya disease. Neuropathology 2000;20:61-4.

9. Scott RM, Smith ER. Moyamoya disease and moyamoya syndrome. N Engl J Med 2009;360:1226-37.

10. Kashiwazaki D, Akioka N, Kuwayama N, et al. Berlin grading system can stratify the onset and predict perioperative complications in adult moyamoya disease. Neurosurgery 2017;81:986-91. 
11. Deng X, Gao F, Zhang D, et al. Direct versus indirect bypasses for adult ischemic-type moyamoya disease: a propensity score-matched analysis. J Neurosurg 2018;128:1785-91.

12. Uchino H, Nakayama N, Kazumata K, et al. Edaravone reduces hyperperfusion-related neurological deficits in adult moyamoya disease: historical control study. Stroke 2016;47:1930-2.

13. Deng X, Gao F, Zhang D, et al. Effects of different surgical modalities on the clinical outcome of patients with moyamoya disease: a prospective cohort study. J Neurosurg 2018;128:1327-37.

14. Kazumata $\mathrm{K}$, Ito $\mathrm{M}$, Tokairin $\mathrm{K}$, et al. The frequency of postoperative stroke in moyamoya disease following combined revascularization: a single-university series and systematic review. J Neurosurg 2014;121:432-40.

15. Zhao M, Deng X, Zhang D, et al. Risk factors for and outcomes of postoperative complications in adult patients with moyamoya disease. J Neurosurg 2018:1-12.

16. Duan L, Bao XY, Yang WZ, et al. Moyamoya disease in China: its clinical features and outcomes. Stroke 2012;43:56-60.

17. Yoshida Y, Yoshimoto T, Shirane R, et al. Clinical course, surgical management, and long-term outcome of moyamoya patients with rebleeding after an episode of intracerebral hemorrhage: an extensive follow-Up study. Stroke 1999;30:2272-6.

18. Liu XJ, Zhang D, Wang S, et al. Clinical features and long-term outcomes of moyamoya disease: a single-center experience with 528 cases in China. J Neurosurg 2015;122:392-9.

19. Kobayashi E, Saeki N, Oishi H, et al. Long-term natural history of hemorrhagic moyamoya disease in 42 patients. J Neurosurg 2000;93:976-80.

20. Fuji K, Ikezaki K, Irikura K, et al. The efficacy of bypass surgery for the patients with hemorrhagic moyamoya disease. Clin Neurol Neurosurg 1997;99(Suppl 2):S194-5.

21. Morioka M, Hamada J, Todaka T, et al. High-risk age for rebleeding in patients with hemorrhagic moyamoya disease: long-term follow-up study. Neurosurgery 2003;52:1049-54. Discussion 54-5.

22. Karasawa J, Kikuchi $\mathrm{H}$, Furuse $\mathrm{S}$, et al. Treatment of moyamoya disease with STA-MCA anastomosis. J Neurosurg 1978;49:679-88.

23. Iwama T, Hashimoto N, Tsukahara T, et al. Superficial temporal artery to anterior cerebral artery direct anastomosis in patients with moyamoya disease. Clin Neurol Neurosurg 1997;99(Suppl 2):S134-6.

24. Ishikawa T, Kamiyama H, Kuroda S, et al. Simultaneous superficial temporal artery to middle cerebral or anterior cerebral artery bypass with pan-synangiosis for Moyamoya disease covering both anterior and middle cerebral artery territories. Neurol Med Chir 2006;46:462-8.

25. Alsop DC, Detre JA, Grossman M. Assessment of cerebral blood flow in Alzheimer's disease by spin-labeled magnetic resonance imaging. Ann Neurol 2000;47:93-100.

26. Chalela JA, Alsop DC, Gonzalez-Atavales JB, et al. Magnetic resonance perfusion imaging in acute ischemic stroke using continuous arterial spin labeling. Stroke 2000;31:680-7.
27. Detre JA, Alsop DC, Vives LR, et al. Noninvasive MRI evaluation of cerebral blood flow in cerebrovascular disease. Neurology 1998;50:633-41.

28. Sugino T, Mikami T, Miyata $\mathrm{K}$, et al. Arterial spin-labeling magnetic resonance imaging after revascularization of moyamoya disease. $J$ Stroke Cerebrovasc Dis 2013;22:811-6.

29. Goetti R, O'Gorman R, Khan N, et al. Arterial spin labelling MRI for assessment of cerebral perfusion in children with moyamoya disease: comparison with dynamic susceptibility contrast MRI. Neuroradiology 2013;55:639-47.

30. Noguchi T, Kawashima M, Irie H, et al. Arterial spin-labeling MR imaging in moyamoya disease compared with SPECT imaging. Eur $J$ Radiol 2011;80:e557-62.

31. Qiu D, Straka M, Zun Z, et al. CBF measurements using multidelay pseudocontinuous and velocity-selective arterial spin labeling in patients with long arterial transit delays: comparison with xenon CT CBF. J Magn Reson Imaging 2012;36:110-9.

32. Feng S, Zhang Y, Sun Z, et al. Application of multimodal navigation together with fluorescein angiography in microsurgical treatment of cerebral arteriovenous malformations. Sci Rep 2017;7:14822.

33. Preidl RH, Schlittenbauer T, Weber M, et al. Assessment of free microvascular flap perfusion by intraoperative fluorescence angiography in craniomaxillofacial surgery. J Craniomaxillofac Surg 2015;43:643-8

34. Okawa M, Abe H, Ogata T, et al. [Efficacy of indocyanine green videoangiography for carotid endarterectomy]. No Shinkei Geka 2012;40:309-17.

35. Ye X, Liu XJ, Ma L, et al. Clinical values of intraoperative indocyanine green fluorescence video angiography with Flow 800 software in cerebrovascular surgery. Chin Med J 2013;126:4232-7.

36. Zipfel GJ, Fox DJ, Rivet DJ. Moyamoya disease in adults: the role of cerebral revascularization. Skull Base 2005;15:27-41.

37. Wang J, Zhang Y, Wolf RL, et al. Amplitude-modulated continuous arterial spin-labeling 3.0-T perfusion MR imaging with a single coil: feasibility study. Radiology 2005;235:218-28.

38. Saida T, Masumoto T, Nakai Y, et al. Moyamoya disease: evaluation of postoperative revascularization using multiphase selective arterial spin labeling MRI. J Comput Assist Tomogr 2012;36:143-9.

39. Wang R, Yu S, Alger JR, et al. Multi-delay arterial spin labeling perfusion MRI in moyamoya disease--comparison with CT perfusion imaging. Eur Radiol 2014;24:1135-44.

40. Gerganov VM, Samii A, Stieglitz L, et al. Typical 3-D localization of tumor remnants of WHO grade II hemispheric gliomas--lessons learned from the use of intraoperative high-field MRI control. Acta Neurochir 2011;153:479-87.

41. Mesiwala AH, Sviri G, Fatemi N, et al. Long-term outcome of superficial temporal artery-middle cerebral artery bypass for patients with moyamoya disease in the US. Neurosurg Focus 2008;24:E15.

42. Fujimura M, Kaneta T, Mugikura S, et al. Temporary neurologic deterioration due to cerebral hyperperfusion after superficial temporal artery-middle cerebral artery anastomosis in patients with adult-onset moyamoya disease. Surg Neurol 2007;67:273-82. 